\title{
Consistent Biped Step Control with COM-ZMP Oscillation Based on Successive Phase Estimation in Dynamics Morphing
}

\author{
Tomomichi Sugihara
}

\begin{abstract}
A self-consistent autonomous foot stepping control is proposed. It works on a self-excited oscillator of COM (center of mass) developed by the author, in which the stabilizability is maximized through a nonlinear feedback to the ZMP (zeromoment point) manipulation. Different from the conventional artificial CPGs (central pattern generators), its dynamics seamlessly morphs from/to that of a standing controller and enables an easy tuning of controller parameters for the desired oscillation amplitude and period. Based on a novel motion index defined by a complex number, the phase and spatial information about the COM-ZMP movement is abstracted. By a successive estimation of the foot-liftable phase, the up-down of feet is controlled so that it automatically synchronizes to the COMZMP oscillation with the unilaterality constraint on the reaction forces explicitly taken into account.
\end{abstract}

\section{INTRODUCTION}

Biped walk is a strongly nonlinear dynamical motion, in which creatures or robots locomote in the world via unstable states through discontinuous deformation of the supporting region by exchanging the stance foot. While the internal space, namely, the joint configuration space is bounded with respect to the joint coordinates, the external space, namely, the world is nearly unbounded. Thus, the stationary walk necessarily becomes a periodic and oscillatory motion which alternates the pivot foot by repeating kicking and swinging of each foot in order to cover wide travel range.

With a biological support that the central pattern generators (CPGs) [1] exist in the spinal cord, various nonlinear oscillators such as coupled van der Pol oscillator [2], Matsuoka oscillator [3] and Kuramoto oscillator [4] have been investigated to build autonomous biped robot controllers [5], [6], [7], [8], [9], [10], [11], [12], [13]. In those studies, the controller first makes an internal body oscillation and results repetitive collisions between the extremities and the environment. Finally, the internal oscillators are expected to be entrained to an external oscillation caused by the collisions. The condition on which that stable entrainment is guaranteed has not been, and will hardly be, clarified. Robot behaviors particularly in transition phase are almost unpredictable in the above scheme due to the absense of a purposeful manipulation of the reaction force. The relationship between the reaction force and the foot motion is not explicitly considered. In this sense, this approach rather

This work was supported in part by Grant-in-Aid for Young Scientists (B) \#20760170, Japan Society for the Promotion of Science and by "The Kyushu University Research Superstar Program (SSP)", based on the budget of Kyushu University allocated under President's initiative.

T. Sugihara (zhidao@ieee.org) is with School of Information Science and Electrical Engineering, Kyushu University, 744 Moto'oka, Nishi-ku, Fukuoka, Japan. depends on luck. Other drawbacks of the above controllers are as follows.

1) They are largely different from the standing regulator in terms of the mathematical structure, so that it is hard, or even impossible, to unify them.

2) The controller comprises several mutually-connected unit oscillators in a bottom-up manner. A tuning policy of a number of controller parameters is hardly found.

3) They are basically nothing more than function generators. Though they have a certain level of adaptability to external perturbations, the stabilization abilities of them are not very high. Extra sensory feedbacks should be superposed on their output signals in order to stabilize the body with respect to the inertial frame. It makes the controller more complicated.

The author[14] have proposed a self-excited oscillation control into which a standing regulator seamlessly morphs by nonlinearly modulating a feedback coefficient. It is based on the manipulation of the zero-moment point (ZMP[15]=center of pressure, COP) to stabilize COM (the center of mass) in which the unilaterality constraint on the reaction forces is explicitly taken into account. A top-down design of the controller enabled easy tunings of the oscillation amplitude and period. The stabilizability condition and its performance limitation have been clarified. A possibility of a biped stepping control which stably synchronizes to the stationary oscillation of COM-ZMP was also proposed by referring the phase information of the limit cycle. The control in transition phase, however, has not been discussed, so that the foot-lifting motion and ZMP movement does not necessarily become consistent due to the lack of spatial information about the ZMP position.

This paper presents a self-consistent biped stepping control. Based on a novel motion index defined by a complex number, the phase and spatial information about the COMZMP movement is abstracted. By a successive estimation of the foot-liftable phase, the up-down control of feet which automatically synchronizes to the COM-ZMP oscillation is achieved with the unilaterality constraint on the reaction forces explicitly taken into account.

\section{UNIFIED REGULATION-OSCILLATION CONTROLLER BASED ON DYNAMICS MORPHING[14]}

Let us consider the biped dynamics in the lateral plane as shown in Fig. 1. Suppose the torque about COM is sufficiently smaller to be neglected than that due to the linear inertial movement of COM about ZMP, and the height of COM $z$ is constant, for simplicity. Also, suppose that the 


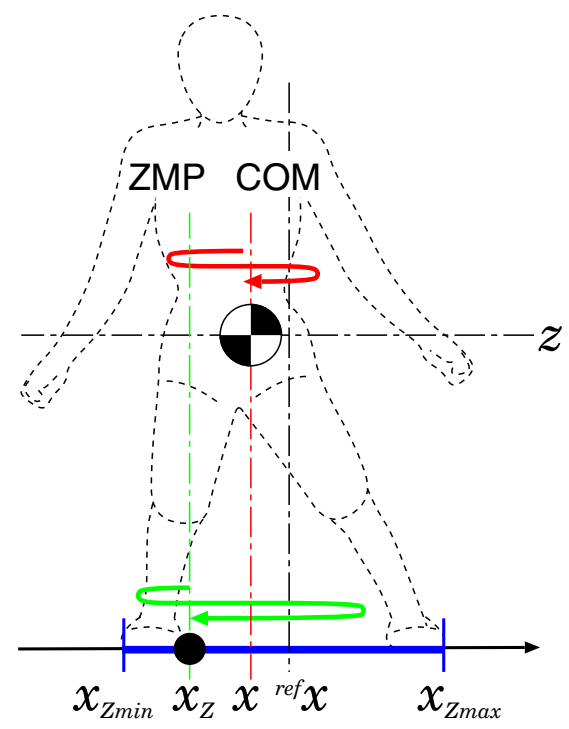

Fig. 1. An approximate mass-concentrated biped model in lateral plane

referential position of COM is at the original point. We get the linear equation of motion as

$$
\ddot{x}=\omega^{2}\left(x-x_{Z}\right),
$$

where $x$ denotes the COM position in sideway, $x_{Z}$ is the ZMP position in sideway, $\omega \equiv \sqrt{g / z}$ and $g=9.8\left[\mathrm{~m} / \mathrm{s}^{2}\right]$ is the acceleration due to the gravity. The ZMP is regarded as the input[16] and is constrained in the supporting region as follows:

$$
x_{Z \min } \leq x_{Z} \leq x_{Z \max },
$$

where $x_{Z \min }$ and $x_{Z \max }$ are the right and left boundaries of the supporting region in $x$-axis, respectively, which satisfy $x_{Z \min }<0<x_{Z \max }$. Even on this simplest model, the control problem would be challenging due to the inequality constraint (2). In our controller, the desired ZMP as the input is designed by a feedback of COM state and the truncation on the edge of the supporting region. Fig. 3 shows phase portraits of the linear feedback with some different pole assignments. Our previous study have revealed that the standing stabilizability shows the best performance under the constraint (2) when one of the two poles of the feedback system coincides with $-\omega$ in case of the linear feedback, and named it the stabilizability-maximized COM-ZMP regulator [17]. Refer the previous report for the details.

In order to emerge a self-excited oscillation, the feedback law is modified to a nonlinear version as follows:

$$
\begin{aligned}
& \tilde{x}_{Z}=(q+1)\left(x+f(\zeta) \frac{\dot{x}}{\omega}\right) \\
& x_{Z}= \begin{cases}x_{Z \max } & \left(\mathrm{S} 1: \tilde{x}_{Z}>x_{Z \max }\right) \\
\tilde{x}_{Z} & \left(\mathrm{~S} 2: x_{Z \min } \leq \tilde{x}_{Z} \leq x_{Z \max }\right) \\
x_{Z \min } & \left(\mathrm{S} 3: \tilde{x}_{Z}<x_{Z \text { min }}\right)\end{cases}
\end{aligned}
$$

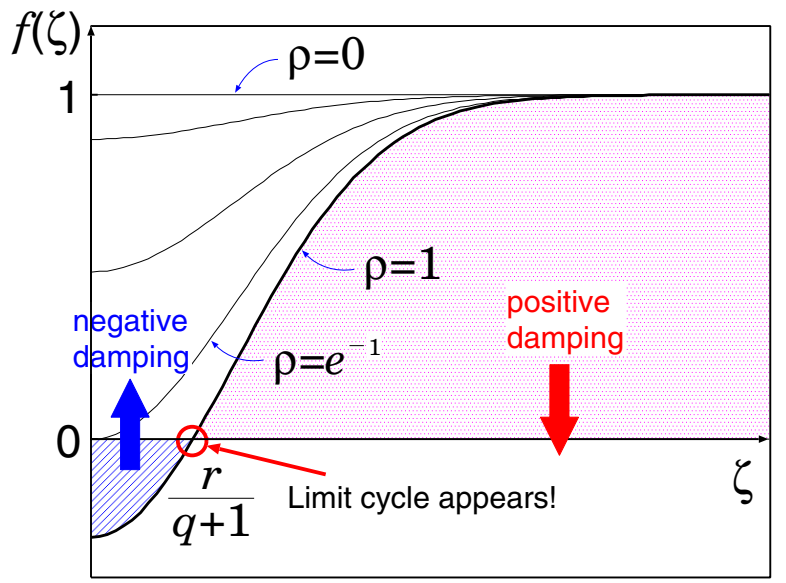

Fig. 2. Damping term of feedback to ZMP is nonlinearly modulated. For arbitrary positive $q, \rho$ and $r, f(\zeta)$ is a monotony increasing function and asymptotically converges to 1 with respect to $\zeta$. At the point where the sign of damping effect is inversed, a stable limit cycle appears.

where

$$
\begin{aligned}
f(\zeta) & \equiv 1-\rho \exp \left\{1-\frac{(q+1)^{2} \zeta^{2}}{r^{2}}\right\}, \\
\zeta & \equiv \sqrt{x^{2}+\frac{\dot{x}^{2}}{\omega^{2} q}},
\end{aligned}
$$

and $q>0, r>0$ and $\rho \geq 0$ are controller parameters. Suppose the actual ZMP is manipulated to follow the above desired ZMP, the dynamics of COM is represented by a piecewise autonomous system as follows:

$$
\ddot{x}= \begin{cases}\omega^{2} x-\omega^{2} x_{Z \max } & (\mathrm{S} 1) \\ -\omega(q+1) f(\zeta) \dot{x}-\omega^{2} q x & (\mathrm{~S} 2) . \\ \omega^{2} x-\omega^{2} x_{Z \min } & (\mathrm{S} 3)\end{cases}
$$

This controller has the following properties.

1) When $\rho=0$, it is identical to the stabilizabilitymaximized COM-ZMP regulator. Namely, a set of the initial conditions from which the COM will stably converge (the stable standing region) in state space is maximized.

2) As $\rho$ gradually increases, the damping term is nonlinearly modulated. When $\rho>e^{-1}$, the system in state (S2) has the following stable ellipsoidal limit cycle

$$
x^{2}+\frac{\dot{x}^{2}}{\omega^{2} q}=\frac{(1+\log \rho) r^{2}}{(q+1)^{2}},
$$

which is a harmonic oscillation with the amplitude $\frac{\sqrt{1+\log \rho} r}{q+1}$ and the period $\frac{2 \pi}{\omega \sqrt{q}}$. Particularly, when $\rho=1$, the amplitude of the oscillation is $\frac{r}{q+1}$. Hence, $q$ and $r$ are decided from the desired period $T$ and amplitude $r^{\prime}$ as

$$
\begin{aligned}
& q=\left(\frac{2 \pi}{\omega T}\right)^{2} \\
& r=(q+1) r^{\prime} .
\end{aligned}
$$



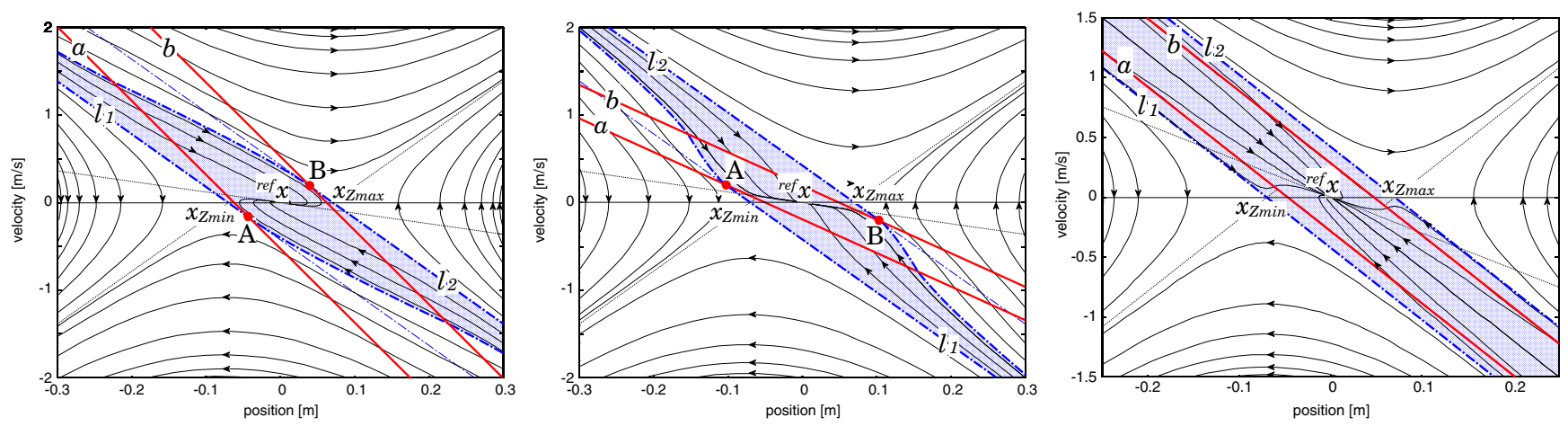

Fig. 3. The assignment of two poles modifies the characteristic of COM-ZMP regulator. In the figure, they are set for $(-0.2 \omega,-0.6 \omega)$ (left), $(-0.2 \omega,-2.0 \omega)$ (center), and $(-0.5 \omega,-\omega)$ (right), respectively, where $\omega=\sqrt{g / 0.27}, x_{Z \text { min }}=-0.07$ and $x_{Z \text { max }}=0.07$ are fixed. The dotted area is the stable standing region. When one of them coincides with $-\omega$, the stable standing region is maximized between two lines $l_{1}$ and $l_{2}$.
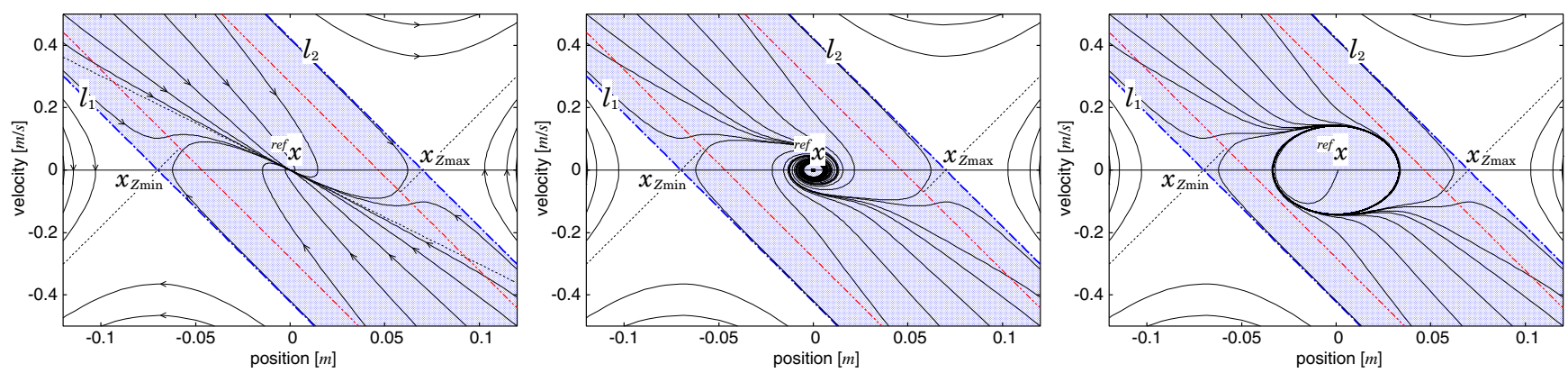

Fig. 4. Dynamics morphing from regulator to oscillator: solution curves of the piecewise autonomous system (7) for (left) $\rho=0$, (center) $\rho=e^{-1}$ and (right) $\rho=1$, where $\omega=\sqrt{g / 0.27},{ }^{r e f} x=0, x_{Z \text { min }}=-0.07, x_{Z \max }=0.07, q=0.5$ and $r=0.05$ are fixed. A stable limit cycle appears when $\rho$ exceeds $e^{-1}$. For any value of $\rho$, the stable standing region stays maximized between two lines $l_{1}$ and $l_{2}$.

3) For arbitrary positive $q, \rho$ and $r, f(\zeta)$ is a monotony increasing function with the following property

$$
\lim _{\zeta \rightarrow \infty} f(\zeta)=1
$$

as Fig. 2 illustrates. Consequently, the controller displays a similar characteristic to the stabilizabilitymaximized COM-ZMP regulator at a distance from the limit cycle.

Fig. 4 shows phase portraits with solution curves of the system (7) for $x_{Z \min }=-0.07[\mathrm{~m}], x_{Z \max }=0.07[\mathrm{~m}]$, $z=0.27[\mathrm{~m}], q=0.5$ and $r=0.05[\mathrm{~m}]$. The left, center and right figures are for $\rho=0, \rho=e^{-1}$ and $\rho=1$, respectively. The stable standing regions are dotted in the figures. In any cases of the above, the stable standing region coincides with a region between two lines $l_{1}$ and $l_{2}$, which is the maximum region in theory. One can see that the system dynamics continually morphs into a self-excited oscillator with the maximized stabilization ability remained.

\section{CONSISTENT STEPPING WITH COM-ZMP OSCILLATION}

The relationship between COM and ZMP in the frequency domain was also studied in our previous report [14]. The transfer function $G(s)$ from $x_{Z}$ to $x$ is

$$
G(s)=\frac{\omega^{2}}{-s^{2}+\omega^{2}} \text {. }
$$

Hence, we get the frequency response

$$
G(i \Omega)=\frac{\omega^{2}}{\Omega^{2}+\omega^{2}}
$$

where $i$ is the imaginary unit. It tells that the phase lag of COM from ZMP is zero and the gain is $|G(i \omega \sqrt{q})|=\frac{1}{q+1}$ when COM oscillates stably. In particular, the amplitude of the ZMP oscillation for $\rho=1$ is $r$ as long as $r<$ $\min \left\{\left|x_{Z \min }\right|,\left|x_{Z \max }\right|\right\}$ is satisfied. In this section, a selfconsistent foot-step controller is designed, based on the phase and spatial information of the COM-ZMP oscillation.

The problem of phase jump (from $2 \pi$ to 0 , for instance) is resolved by introducing a complex number. An idea to define the system phase based on the behavior of ZMP was proposed by Morimoto et al.[13], which is represented by a complex number as follows:

$$
{ }^{M} p_{Z} \equiv x_{Z}-\frac{\dot{x}_{Z}}{\omega \sqrt{q}} i
$$

where the imaginary part is scaled so that the limit cycle of ${ }^{M} p_{Z}$ forms a circle in complex plane. However, ZMP depends on the equilibrium of torques, and thus, it's rate $\dot{x}_{Z}$ is sensitive and varies discontinuously when jumps or saturations of ZMP occur. In order to avoid this problem, let 


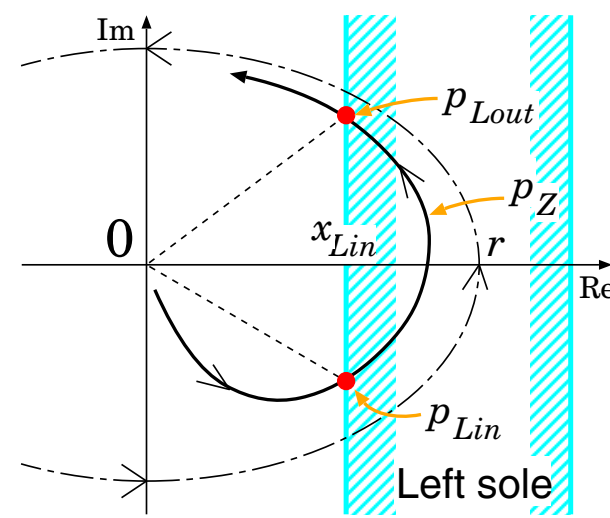

Fig. 5. Locus of $p_{Z}$ in transition phase. The intersection of it and the left sole is segmented by $p_{\text {Lin }}$ and $p_{\text {Lout }}$, the real parts of which are $x_{L i n}$ The right foot is liftable when $p_{Z}$ is on the segment. us define the system phase $p_{Z}$ as follows:

$$
p_{Z} \equiv x_{Z}-\frac{(q+1) \dot{x}}{\omega \sqrt{q}} i .
$$

Suppose the inner side of the left sole in $x$-axis is located at $x_{\text {Lin }}$. Let us consider the locus of $p_{Z}$ as Fig. 5. The intersection of the locus and the left sole is segmented by two points $p_{\text {Lin }}$ and $p_{\text {Lout }}$, the real parts of which are both $x_{L i n}$. The right foot is liftable when $p_{Z}$ is on this segment. An idea to control the up-down of the right foot is to detach off the ground at $p_{Z}=p_{L i n}$ and touch down at $p_{Z}=p_{\text {Lout }}$. The problem is, however, that it is impossible to know those two points in advance. Then, we successively estimate these values as $\bar{p}_{\text {Lin }}$ and $\bar{p}_{\text {Lout }}$, which are computed only when $\left|p_{Z}\right|>x_{L i n}$ is satisfied as follows:

$$
\begin{gathered}
\bar{p}_{\text {Lin }}=x_{\text {Lin }}-\sqrt{\left|p_{Z}\right|^{2}-x_{L i n}^{2}} i \\
\bar{p}_{\text {Lout }}=x_{\text {Lin }}+\sqrt{\left|p_{Z}\right|^{2}-x_{L i n}^{2}} i .
\end{gathered}
$$

As COM converges to the limit cycle, they also asymptotically converge to the start and end points of the steady oscillation, respectively, as Fig. 6 depicts. More importantly, it is guaranteed that ZMP lies within the left sole as long as $p_{Z}$ satisfies

$$
0<\phi_{L}<1
$$

where

$$
\phi_{L} \equiv \frac{L p_{Z} / \bar{p}_{\text {Lin }}}{L \bar{p}_{\text {Lout }} / \bar{p}_{\text {Lin }}} .
$$

In regular situations, $\phi_{L}$ increases from 0 to 1 smoothly during the oscillation. Hence, $\phi_{L}$ is expected to work as the phase information for the foot-lifting. Only when $\left|p_{Z}\right|>$ $x_{L i n}$ and condition (18) are satisfied, we define the lifting height of the right foot $z_{R}$ with respect to $\phi_{L}$ as

$$
z_{R}=\frac{1}{2} \frac{h\left|p_{Z}\right|}{r} \sigma(\rho)\left(1-\cos 2 \pi \phi_{L}\right)
$$
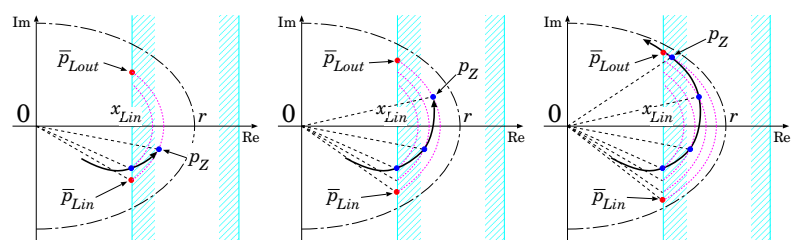

Fig. 6. The start and end points of the intersecting segment of $p_{Z}$ locus and the sole are successively

Fig. 6. The start and end points of the intersecting segment of $p_{Z}$ locus and the sole are successively estimated as $\bar{p}_{\text {Lin }}$ and $\bar{p}_{\text {Lout }}$. In the course of convergence to the limit cycle, they automatically
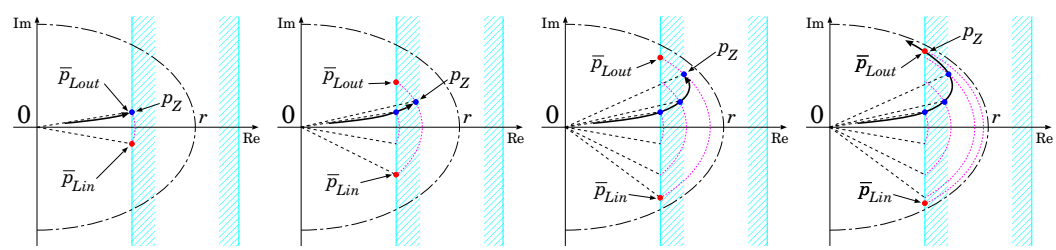

in the first quadrant of complex plane, the foot-step is consistently controlled since $z_{R}$ is symmetric with respect to $\phi_{L}$.

where $h$ is a constant for the nominal lifting height, and $\sigma(\rho)$ is defined as

$$
\sigma(\rho) \equiv \begin{cases}1 & (\rho>1) \\ \frac{\rho e-1}{e-1} & \left(e^{-1} \leq \rho \leq 1\right) \\ 0 & \left(0 \leq \rho<e^{-1}\right)\end{cases}
$$

Otherwise, we let $z_{R}=0$. The maximum lifting height depends on $\rho$ and $\left|p_{Z}\right| . \rho$ means the degree of self-excitation, while $\left|p_{Z}\right| / r$ measures the degree of convergence to the limit cycle; the larger both of them becomes, the more the stepping motion is activated. The lifting height of the left foot $z_{L}$ is also defined in a symmetric way. By using this technique, the up-down motion of feet automatically synchronizes to ZMP without the information of the actual $p_{\text {Lin }}$ and $p_{\text {Lout }}$, which we cannot know during the motion.

Note that $\phi_{L}$ does not necessarily monotonically increase. While the real part of $p_{Z}$ is ZMP, its imaginary part refers the COM velocity. This difference possibly causes strange situations where both the start and end points of the intersection of $p_{Z}$ and the left sole lie in the first quadrant of complex plane as Fig. 7 shows. At the start point, $p_{Z}$ is not regarded as at $p_{\text {Lin }}$ but at $p_{\text {Lout }}$. However, this doesn't violate consistency of the stepping motion since $z_{R}$ is symmetric and continuous with respect to $\phi_{L}=0 \sim 1$.

\section{Simulation}

An autonomous stepping motion is simulated based on the dynamical model of a miniature anthropomorphic robot mighty[18]. It is assumed that the total mass is concentrated at COM, for simplicity. Two cases where $r=0.05[\mathrm{~m}]$ and $r=0.09[\mathrm{~m}]$ were conducted with $x_{Z \min }=-0.0845[\mathrm{~m}]$, $x_{Z \max }=0.0845[\mathrm{~m}], z=0.27[\mathrm{~m}], h=0.02[\mathrm{~m}]$ and $q=$ 0.5. $\rho$ is defined as the function of time as

$$
\rho=\left\{\begin{array}{ll}
\frac{t}{T_{1}} & \left(0 \leq t<T_{1}=0.2[\mathrm{~s}]\right) \\
1 & \left(T_{1} \leq t<T_{2}=4.8[\mathrm{~s}]\right) \\
\frac{T-t}{T-T_{2}} & \left(T_{2} \leq t<T=5.0[\mathrm{~s}]\right) \\
0 & (t \geq T)
\end{array},\right.
$$



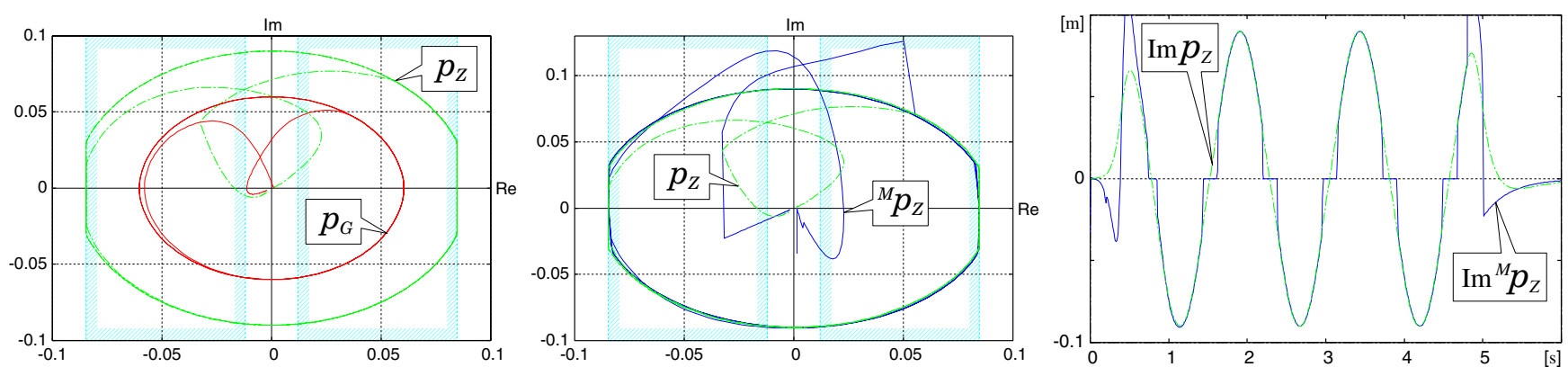

Fig. 8. (Left) Loci of COM and ZMP phases in the tested motion for $r=0.09$ (Center) Comparison between $p_{Z}$ and ${ }^{M} p_{Z}$ (Right) Comparison of the time series of imaginary parts of $p_{Z}$ and ${ }^{M} p_{Z}$. Since $r>\left|x_{Z \text { max }}\right|=\left|x_{Z \text { max }}\right|$, ZMP is frequently saturated at the edge of the supporting region.

by which it is expected that the robot motion continually switches from the steady stance to the oscillation, and back from the oscillation to the steady stance again.

The left side of Fig. 8 shows loci of $p_{Z}$ and another complex number

$$
p_{G} \equiv x-\frac{\dot{x}}{\omega \sqrt{q}} i
$$

which visualizes the COM movement in complex plane for $r=0.09$. While they are far from the ellipsoidal limit cycle in transition phase, they smoothly converge to the limit cycles of each. Since $r>\left|x_{Z \text { min }}\right|=\left|x_{Z \max }\right|$, ZMP was frequently saturated at the edge of the supporting region, so that the limit cycle of $p_{Z}$ is distorted. Hatched regions are for the left and right soles. For reference, ${ }^{M} p_{Z}$ and $p_{Z}$ are plotted in the center of Fig. 8. Time series of their imaginary parts are also plotted in the right of Fig. 8. One can see that ${ }^{M} p_{Z}$ largely jumps at each juncture and the phase often varies discontinuously. Hence, the proposed definition of the phase is preferable rather than Morimoto et al.'s.

Figs. 9 and 10 show the time series of $\rho, x, x_{Z}, z_{L}, z_{R}, \phi_{L}$ and $\phi_{R}$ in case of $r=0.05$ and $r=0.09$, respectively. The supporting region is dotted in the graphs of $x$ and $x_{Z}$. While the period of the stationary oscillation for $\rho=1$ coincides with its theoretical value $\simeq 1.47[\mathrm{~s}]$ in the former case, the presence of uncontrollable phases due to the saturations of ZMP in the latter case made the period of the stationary oscillation longer than $1.47[\mathrm{~s}]$. In spite of this difference of period, the behaviors of the feet are consistent in any cases.

$\phi_{R}$ started from 1, was decreased and was again increased to 1 in transition phase of both cases (around $t \simeq 0.3$ in the former case and $t \simeq 0.3 \sim 0.5$ in the latter case), which means that $p_{Z}$ crossed $x_{L i n}$ twice in the first quatient in this term. As explained in the previous section, it didn't affect badly to the motion. Although $\rho$ was varied according to Eq.(22) without any care about the supporting region, selfconsistent biped stepping motions with COM and ZMP were automatically emerged.

\section{CONCLUSION}

A self-consistent biped stepping control on the unified regulation-oscillation control by dynamics morphing was proposed. A novel motion index and successive estimation of the foot-liftable segment enabled an automatic synchronization of foot-step and COM-ZMP oscillation with the unilaterality constraint on the reaction forces satisfied.

The proposed control scheme suggests a possibility to maneuver the biped stepping only by modulating the degree of self-excitation $\rho$, which will be a fundamental technique for a completely autonomous controller design.

\section{REFERENCES}

[1] S. Grillner, "Locomotion in vertebrates: Central mechanisms and reflex Interaction," Physiological Reviews, vol. 55, pp. 367-371, 1975.

[2] D. A. Linkens, "The stability of entrainment conditions for RLC compled van der Pol oscillators used as a model for intestinal electrical rhythms," Bulletin of Mathematical Biology, vol. 39, no. 3, pp. 359372, 1977.

[3] K. Matsuoka, "Mechanisms of frequency and pattern control in the neural rhythm generators," Biological Cybernetics, vol. 56, pp. 345353, 1987.

[4] Y. Kuramoto, Self-entrainment of a population of coupled non-linear oscillators, ser. Lecture Notes in Physics. Springer, 1975, pp. 420422.

[5] R. Kato and M. Mori, "Control Method of Biped Locomotion Giving Asymptotic Stability of Trajectory," Automatica, vol. 20, no. 4, pp. 405-414, 1984.

[6] L. Jalics, H. Hemami, and Y. F. Zheng, "Pattern Generation Using Coupled Oscillators for Robotic and Biorobotic Adaptive Periodic Movement," in Proceedings of the 1997 IEEE International Conference on Robotics \& Automation, 1997, pp. 179-184.

[7] M. S. Dutra, A. C. de Pina Filho, and V. F. Romano, "Modeling of a bipedal locomotor using coupled nonlinear oscillators of Van der Pol," Biological Cybernetics, vol. 88, pp. 286-292, 2003.

[8] R. Héliot and B. Espiau, "Multisensor Input for CPG-Based SensoryMotor Coordination," IEEE Transactions on Robotics, vol. 24, no. 1, pp. 191-195, 2008.

[9] G. Taga, "A model of the neuro-musculo-skeletal system for human locomotion," Biological Cybernetics, vol. 73, pp. 97-121, 1995.

[10] O. Katayama, Y. Kurematsu, and S. Kitamura, "Theoretical Studies on Neuro Oscillator for Application of Biped Locomotion," in Proceedings of the 1995 IEEE International Conference on Robotics \& Automation, 1995, pp. 2871-2876.

[11] S. Miyakoshi, G. Taga, Y. Kuniyoshi, and A. Nagakubo, "Three Dimensional Bipedal Stepping Motion using Neural Oscillators Towards Humanoid Motion in the Real World," in Proceedings of the 1998 IEEE/RSJ Internatinal Conference on Intelligent Robots and Systems, 1998, pp. 84-89.

[12] S. Aoi and K. Tsuchiya, "Stability Analysis of a Simple Walking Model Driven by an Oscillator With a Phase Reset Using Sensory Feedback," IEEE Transactions on Robotics, vol. 22, no. 2, pp. 391397, 2006.

[13] J. Morimoto, G. Endo, J. Nakanishi, and G. Cheng, "A Biologically Inspired Biped Locomotion Strategy for Humanoid Robots: Modulation of Sinusoidal Patterns by a Coupled Oscillator Model," IEEE Transactions on Robotics, vol. 24, no. 1, pp. 185-191, 2008. 

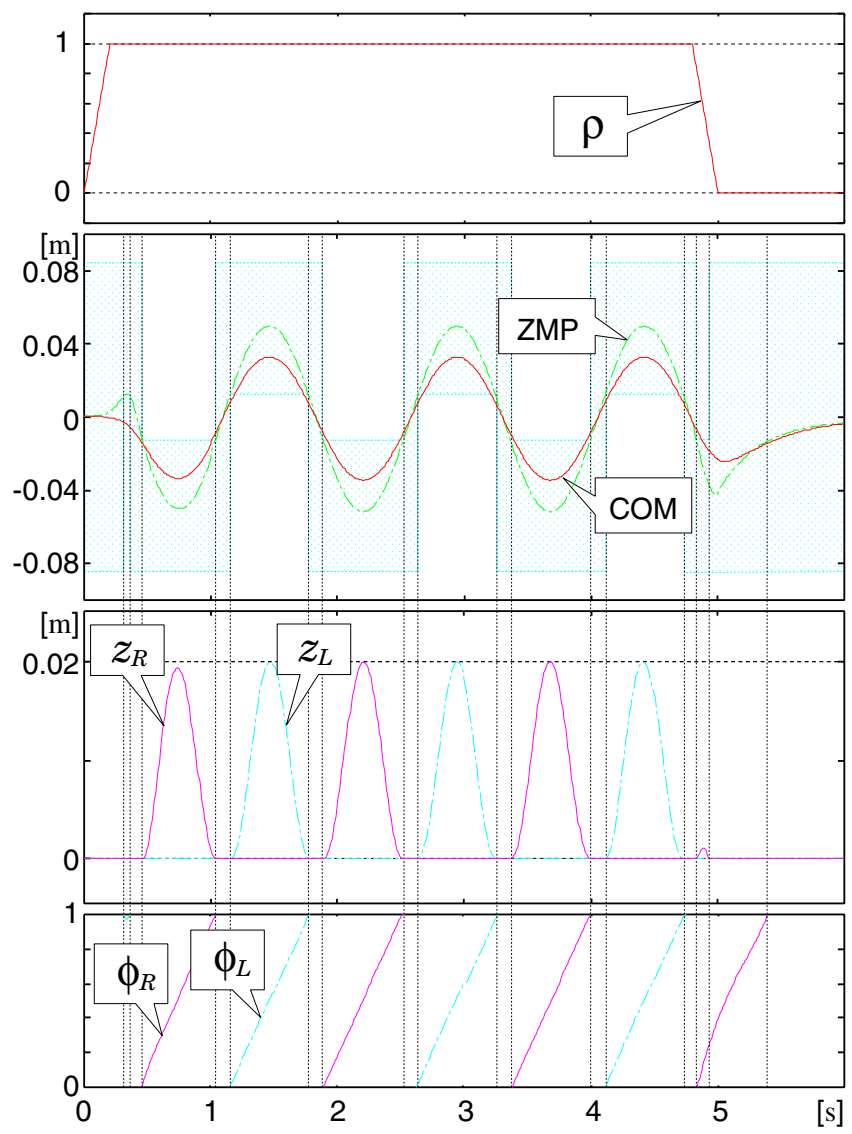

Fig. 9. Loci of $\rho$, COM, ZMP, feet and foot-lifting phase in the tested motion for $r=0.05$. Even though $\rho$ was varied without any care about the supporting region, the feet were consistently controlled with the behaviors of COM and ZMP
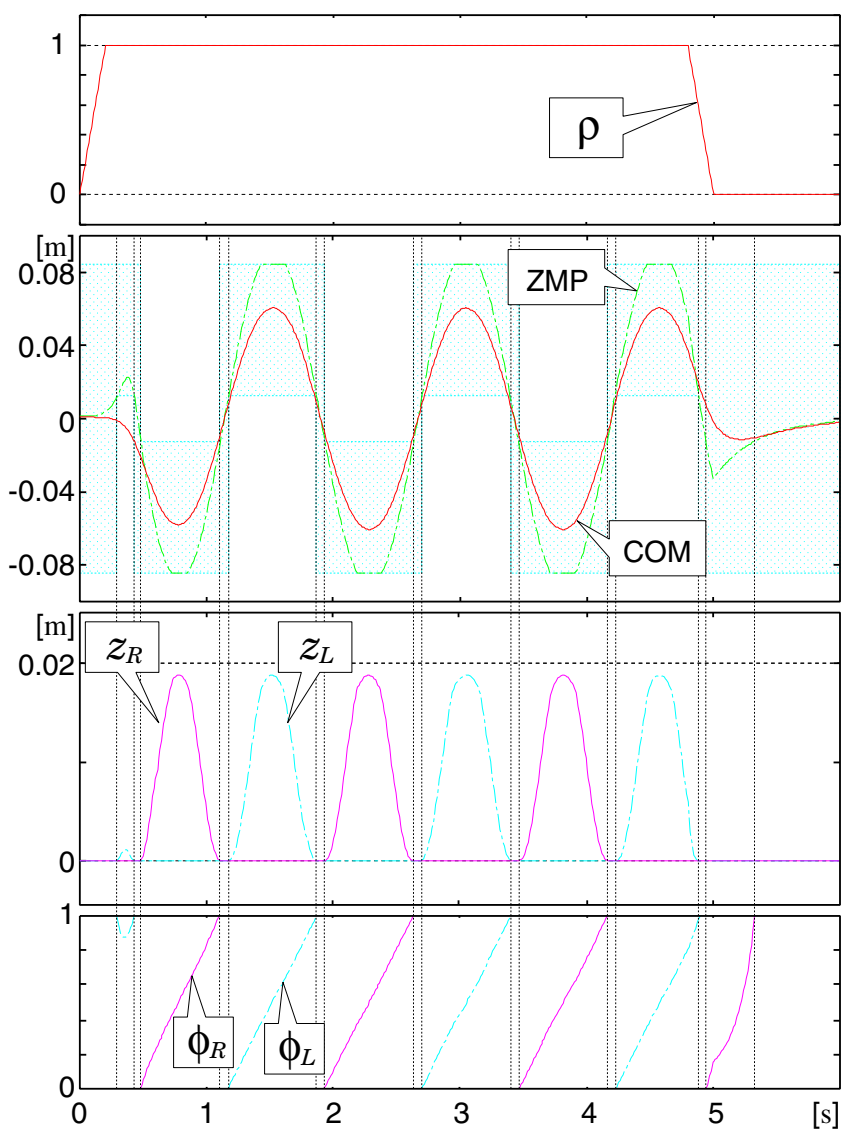

Fig. 10. Loci of $\rho$, COM, ZMP, feet and foot-lifting phase in the tested motion for $r=0.09$. Since $r>\left|x_{Z \text { min }}\right|=\left|x_{Z \text { max }}\right|$, ZMP was frequently saturated at the edge of the supporting region. The feet were still consistently controlled.
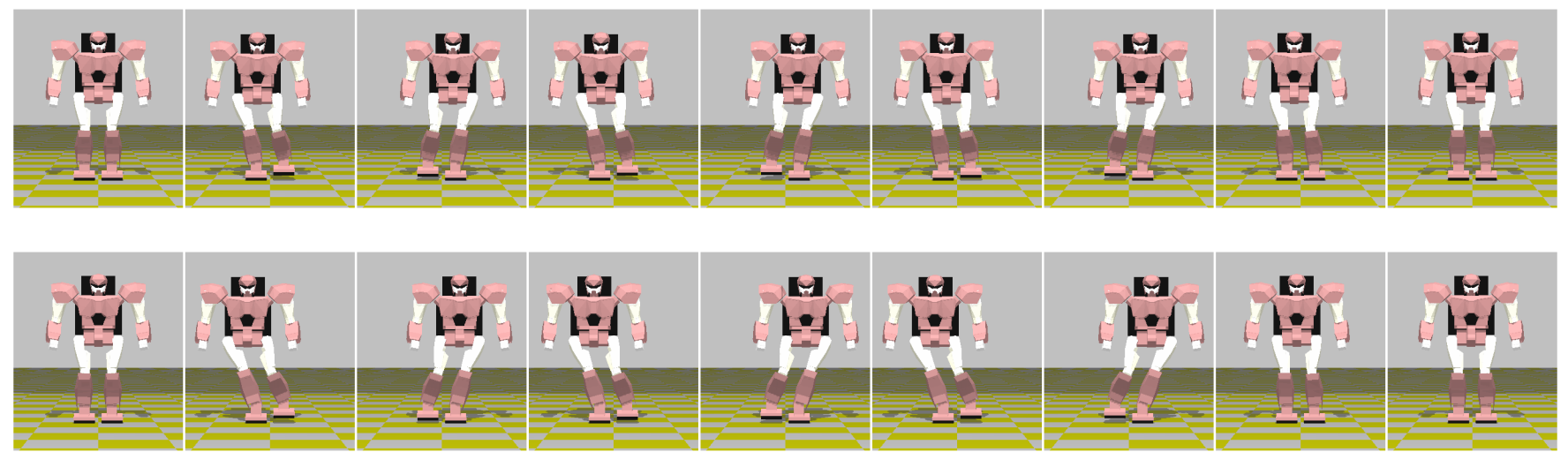

Fig. 11. Snapshots of the tested motion for $r=0.05$ (upper) and $r=0.09$ (lower). The robot motion is seamlessly controlled from the standing via the stepping to the standing again only by the modulation of $\rho$.

[14] T. Sugihara, "Dynamics Morphing from Regulator to Oscillator on Bipedal Control," in Proceedings of the 2009 IEEE/RSJ Internatinal Conference on Intelligent Robots and Systems, 2009 (to appear).

[15] M. Vukobratović and J. Stepanenko, "On the Stability of Anthropomorphic Systems," Mathematical Biosciences, vol. 15, no. 1, pp. 1-37, 1972.

[16] K. Mitobe, G. Capi, and Y. Nasu, "Control of walking robots based on manipulation of the zero moment point," Robotica, vol. 18, pp.
651-657, 2000.

[17] T. Sugihara, "Standing Stabilizability and Stepping Maneuver in Planar Bipedalism based on the Best COM-ZMP Regulator," in Proceedings of the 2009 IEEE International Conference on Robotics \& Automation, 2009, pp. 1966-1971.

[18] T. Sugihara, K. Yamamoto, and Y. Nakamura, "Hardware design of high performance miniature anthropomorphic robots," Robotics and Autonomous System, vol. 56, pp. 82-94, 2007. 